\title{
THE ORGANISING COMMITTEE
}

V. A. Ambartsumian, E. M. Burbidge, R. H. Dicke, E. B. Holmberg, I. D. Novikov, M. Ryle, M. Schmidt, D. W. Sciama, J. Smak, Ya. B. Zel'dovich (Chairman),

A. Zieba, W. Zonn, and M. S. Longair (Secretary)

\section{LOCAL ORGANISING COMMITTEE}

K. Rudnicki, F. Skolimowska, and A. Zieba 